\title{
A Survey of Fuzzy Based Image Denoising Techniques
}

\author{
Mansi Pathak ${ }^{1}$,Dr.G.R.Sinha ${ }^{2}$ \\ (1 Deptt.of ET\&T, SSCET College, Research Scholar / CSVTU University, Bhilai, C.G., India) \\ (2 Professor(EC) \& Associate Director, SSCET College / CSVTU University, Bhilai, C.G., India)
}

\begin{abstract}
Image denoising algorithms may be the oldest in image processing. A first pre-processing step in analyzing such datasets is denoising, that is, estimating the unknown signal of interest from the available noisy data. There are several different approaches to denoise images. To remove noise several techniques and image denoising filters are used. This paper shows a comparative study and analysis of image denoising techniques relying on fuzzy filters.
\end{abstract}

Keywords: Image Denoising ,Fuzzy Filters, Fuzzy Logic

\section{INTRODUCTION}

Whenever an image is converted from one form to another, such as, digitizing, scanning, transmitting, storing, etc., some degradation occurs at the output. Hence, the output image has to undergo a process called image enhancement. Fuzzy image processing is the collection of all approaches that understand, represent and process the images, their segments and features as fuzzy sets. The representation and processing depend on the selected fuzzy technique and on the problem to be solved. The idea of fuzzy sets is simple and natural. For instance, we want to define a set of gray levels that share the property dark. In classical set theory, we have to determine a threshold, say the gray level 100. All gray levels between 0 and 100 are element of this set: the others do not belong to the set. But the darkness is a matter of degree. So, a fuzzy set can model this property much better. The basis for fuzzy logic is the basis for human communication. This observation underpins many of the other statements about fuzzy logic. Because fuzzy logic is built on the structures of qualitative description used in everyday language, fuzzy logic is easy to use. A filtering system needs to be capable of reasoning with vague and uncertain informatioa this suggests the use of fuzzy logic.

\section{Survey OF FuZzy BaSed IMAge DenOISING TeChNiQues}

The fundamental problem of image and signal processing is to effectively reduce noise from a digital image while keeping its features intact (e.g.. edges. colour component distances. etc). The nature of the noise removal problem depends on the type of the noise corrupting the image.

\section{Noise in an image}

The two most commonly occurring types of noise are

(i) Additive noise (e.g. Gaussian and Impulse noise)

(ii) Multiplicative noise (e.g. Speckle noise).

Impulse noise is usually characterized by some portion of image pixels that are corrupted, leaving the remaining pixels unchanged. Examples of impulse noise are fixed-valued impulse noise and randomly valued impulse noise. We talk about additive noise when a value from a certain distribution is added to each image pixel, for example, a Gaussian distribution. Multiplicative noise is generally more difficult to remove from images than additive noise because the intensity of the noise varies with the signal intensity (e.g. speckle noise).In tile literature several (fuzzy and non-fuzzy filters have been studied for impulse noise reduction. Impulse noise is caused by errors in the data transmission generated in noisy sensors or communication channels, or by errors during tile data capture from digital cameras. Noise usually quantified by the percentage of pixels which are corrupted. Corrupted pixels are either set to the maximum value or have single bits flipped over. In some cases, single pixels are set alternatively to zero or to the maximum value. This is the most common form of impulse noise and is called salt and pepper noise. Noise smoothing and edge enhancement are inherently conflicting processes, since smoothing a region might destroy an edge, while sharpening edges might lead to unnecessary noise .

\section{Fuzzy Filters}


Noise reduction is an important area for image processing. Besides classical filters, there are lots of fuzzy filters in the literature. Images can be corrupted with impulse noise, Gaussian noise or both. Depending on the type of noise, filters can be used. The fuzzy filters are categorized into two subclasses :

(a) Fuzzy-classical filters: Fuzzy Classical filters are filters that use fuzzy logic and these are the modification of the classical filters. Some of the fuzzy- classical filters are

(i) Fuzzy median filter- Fuzzy median filter is well known for removing impulse noise. It is the fuzzy rank ordering of samples and is simply a replacement of conventional median filter with fuzzy counterparts.

(ii) Fuzzy impulse noise detection and reduction method- this filter by Selhulte detects the impulse noise and any other noise in the image. It contains the noise - detection step and filtering step to preserve the edges. Fuzzy detection step uses fuzzy gradient values in eight directions with a $3 \times 3$ window, which indicates the degree of central pixel as an impulse noise pixel. A fuzzy set is constructed based on the gradient.

(b) Fuzzy filters: These are filters that are totally dependent on fuzzy logic and they do not have any connection with classical filters. A few fuzzy filters ale discussed below.

(i) Gaussian noise reduction filter (GOA) - This filter is specially designed to remove Gaussian noise. Averaging is done for a pixel using other neighborhood pixels and simultaneously taking care of the other image structures such as edges. To achieve this, two features are required. First, in order to distinguish between the variations die to noise and the image structures, the filter uses gradient for all the eight directions. Second, the membership functions are adapted accordingly to the noise level to perform fuzzy smoothing. The filter is applied iteratively.

(ii) Histogram adaptive filter (HAF) - This type of filter removes high impulsive noise, preserving edge information. In HAF, each input pixel is considered a fuzzy variable and a square window of size $3 \mathrm{X} 3$ is sided over the entire image and the filter output is associated with each centre pixel in a window. Three fuzzy sets for dark, Medium, and bright are created and the membership functions for these fuzzy sets are calculated. Then fuzzy inference rules based on the Takagi-Sugeno approach with a slight difference is used in a final output decision process.

Image denoising still remains a challenge for researchers because noise removal introduces artifacts and causes blurring of the images. This paper describes different methodologies for noise reduction giving an insight as to which filter should be used to find the most reliable estimate of the original image data analysis of some methods that are available in the literature is given in below section.

\subsection{Fuzzy impulse noise detection and reduction method (FIDRM) [1] and noise adaptive fuzzy switching median filter for salt and pepper noise reduction (NAFSM)}

\section{FIDRM:-}

The FIDRM filter is a two step filter. First is the detection step which uses fuzzy rules to know that the pixel is corrupted with impulse noise or not.

\section{A. DETECTION STEP}

A 3 X 3 neighbourhood window is used for each pixel $(i, j)$ of the image (not a border pixel). If $A$ is the input image, then we define the gradient $\nabla_{(k, 1)} \mathrm{A}(\mathrm{i}, \mathrm{j})$ as the difference

$$
\nabla_{(k, 1)} A(i, j)=A(i+k, j+1)-A(i, j) \text { with } k, 1\{-1,0,1\}
$$

Where $(i, j)$ is the center of the gradient and pair $(k, l)$ corresponds to one of the eight directions. So the eight gradient values corresponding to the eight directions are called basic gradients. As shown in the table, there is one basic and two related gradients corresponding to each direction. The two related gradients in the same direction are determined by the centres making a right angle with the direction of the basic gradient

TABLE 1 INVOLVED GRADIENT VALUES

\begin{tabular}{|c|c|c|}
\hline $\mathbf{R}$ & $\begin{array}{l}\text { BASIC } \\
\text { gradient }\end{array}$ & RELATED GRADIENT \\
\hline $\begin{array}{l}\mathbf{N} \\
\mathbf{W}\end{array}$ & $\nabla_{(\mathrm{NW}) \mathrm{A}(\mathrm{i}, \mathrm{j})}$ & $\nabla_{(\mathrm{NW})} \mathrm{A}(\mathrm{i}+1, \mathrm{j}-1), \nabla_{(\mathrm{NW})} \mathrm{A}(\mathrm{i}-1, \mathrm{j}+1)$ \\
\hline
\end{tabular}




\begin{tabular}{|c|c|c|}
\hline $\mathbf{N}$ & $\nabla_{(\mathrm{N})} \mathrm{A}(\mathrm{i}, \mathrm{j})$ & $\nabla_{(\mathrm{N})} \mathrm{A}(\mathrm{i}, \mathrm{j}-1), \nabla_{(\mathrm{N})} \mathrm{A}(\mathrm{i}, \mathrm{j}+1)$ \\
\hline $\begin{array}{l}\mathbf{N} \\
\mathbf{E}\end{array}$ & $\nabla_{(\mathrm{NE})} \mathrm{A}(\mathrm{i}, \mathrm{j})$ & $\nabla_{(\mathrm{NE})} \mathrm{A}(\mathrm{i}-1, \mathrm{j}-1), \nabla_{(\mathrm{NE}) \mathrm{A}(\mathrm{i}+1, \mathrm{j}+1)}$ \\
\hline $\mathbf{E}$ & $\nabla_{(\mathrm{E}) \mathrm{A}(\mathrm{i}, \mathrm{j})}$ & $\nabla_{(\mathrm{E})} \mathrm{A}(\mathrm{i}-1, \mathrm{j}), \nabla_{(\mathrm{E})} \mathrm{A}(\mathrm{i}+1, \mathrm{j})$ \\
\hline $\begin{array}{l}\mathbf{S} \\
\mathbf{E}\end{array}$ & $\nabla_{(\mathrm{SE}) \mathrm{A}(\mathrm{i}, \mathrm{j})}$ & $\nabla_{(\mathrm{SE})} \mathrm{A}(\mathrm{i}-1, \mathrm{j}+1), \nabla_{(\mathrm{SE})} \mathrm{A}(\mathrm{i}+1, \mathrm{j}-1)$ \\
\hline $\mathbf{S}$ & $\nabla_{(\mathrm{s}) \mathrm{A}(\mathrm{i}, \mathrm{j})}$ & $\nabla_{(\mathrm{s}) \mathrm{A}(\mathrm{i}, \mathrm{j}-1)}, \nabla_{(\mathrm{s}) \mathrm{A}(\mathrm{i}, \mathrm{j}+1)}$ \\
\hline $\begin{array}{l}\mathbf{S} \\
\mathbf{W}\end{array}$ & $\nabla_{(\mathrm{sw}) \mathrm{A}(\mathrm{i}, \mathrm{j})}$ & $\nabla_{(\mathrm{sw})} \mathrm{A}(\mathrm{i}-1, \mathrm{j}-1), \nabla_{(\mathrm{sw}) \mathrm{A}(\mathrm{i}+1, \mathrm{j}+1)}$ \\
\hline $\mathbf{W}$ & $\nabla_{(\mathrm{W}) \mathrm{A}(\mathrm{i}, \mathrm{j})}$ & $\nabla_{(\mathrm{w})} \mathrm{A}(\mathrm{i}-1, \mathrm{j}), \nabla_{(\mathrm{w}) \mathrm{A}(\mathrm{i}+1, \mathrm{j})}$ \\
\hline
\end{tabular}

Now the fuzzy gradient value $\left.\right|_{R} ^{F} A(i, j) \mid$ for each of the eight directions is calculated by the following fuzzy rule:

If $\left|\nabla_{R A}(\mathrm{i}, \mathrm{j})\right|$ is large AND $\left|\nabla^{\prime}{ }_{R A}(\mathrm{i}, \mathrm{j})\right|$ is small OR

If $\left|\nabla^{\prime}{ }_{R A} A(i, j)\right|$ is large AND $\left|\nabla^{*}{ }_{R A} A(i, j)\right|$ is small OR

$\nabla_{\mathrm{RA}} \mathrm{A}(\mathrm{i}, \mathrm{j})$ is big positive AND $\nabla^{\prime}{ }_{\mathrm{R}} \mathrm{A}(\mathrm{i}, \mathrm{j})$ AND $\nabla^{*}{ }_{\mathrm{R}} \mathrm{A}(\mathrm{i}, \mathrm{j})$ are big negative OR

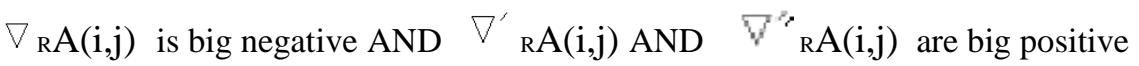

THEN $\left|\nabla_{\mathrm{R}}^{\mathrm{F}} \mathrm{A}(\mathrm{i}, \mathrm{j})\right|$ is large.

Where $\nabla_{R} A(i, j)$ is the basic gradient value and $\nabla^{\prime}{ }_{R A} A(i, j)$ is the first related gradient and $\nabla^{{ }^{r}}{ }_{R A} A(i, j)$ is the second related gradient. The terms "large", "small", "big negative" and "big positive" can be represented as fuzzy sets. Fuzzy sets are represented by a membership function as shown in figure.

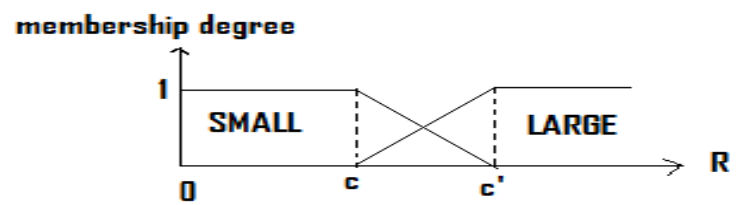

(a)

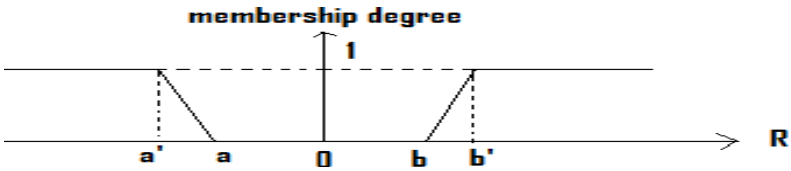

(b)

Figure 1 Membership functions (a) SMALL respectively, LARGE (b) BIG NEGATIVE, respectively, BIG POSITIVE

To determine if a central pixel (a nonborder pixel) is an impulse noise pixel, the following fuzzy rule is employed:

IF most of the eight $\nabla_{\mathrm{RA}}(\mathrm{i}, \mathrm{j})$ are large THEN the central pixel is an impulse noise pixel.

If a pixel $(\mathrm{i}, \mathrm{j})$ is detected as an impulse noise pixel, then the corresponding grayscale value is stored in a histogram

\section{B. FILTERING STEP}

Now, calculate five peak values in the noise histogram i.e. the corresponding gray scale value where maximum is reached ( five $\mathrm{p}_{\mathrm{K}}$ ) and four parameters which are used to construct the fuzzy set more or less impulse noise.

$$
\begin{aligned}
& \mathrm{a}_{\mathrm{k}}=\mathrm{p}_{\mathrm{k}}-\mathrm{THR}_{\mathrm{a}}, \mathrm{b}_{\mathrm{k}}==\mathrm{p}_{\mathrm{k}}-\mathrm{THR} \mathrm{k} \\
& \mathrm{c}_{\mathrm{k}}=\mathrm{p}_{\mathrm{k}}+\mathrm{THR}_{\mathrm{c}}, \mathrm{d}_{\mathrm{k}}=\mathrm{p}_{\mathrm{k}}+\mathrm{THR} \mathrm{R}
\end{aligned}
$$




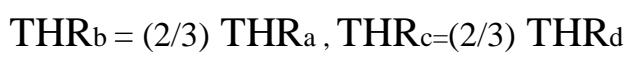

and

$\mathrm{THRa}=\mathrm{THRd}, \min (25,[\sigma])$ where$[\sigma]$ is the largest integer value smaller than the variance. These four parameters are used to construct the fuzzy set more or less impulse noise as shown in figure below

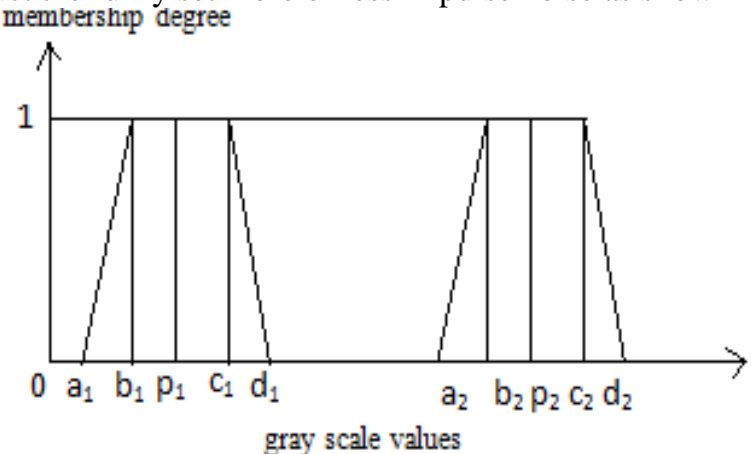

Figure 2 Membership function representing the fuzzy set "more or less impulse noise".

\section{NAFSM:-}

The NAFSM filter is a recursive, two stage filter. The first stage is the detection stage, it will detect the intensities of salt and pepper noise.

\section{A. Detection Stage}

In the detection stage the two peak intensities in noise histogram are searched, i.e. the two local maximums, Lsalt and Lpepper. Then a binary noise mask N(i,j) will be created to mark the location of "noise pixels" by using the equation

$\mathrm{N}(\mathrm{i}, \mathrm{j})=$

$$
0, X(I, j)=\text { Lsalt or Lpepper }
$$

1, otherwise

Where $X(i, j)$ stands for the pixel at location $(i, j)$ with intensity $X$.

\section{B. Filtering Stage}

After the creation of binary noise mask $\mathrm{N}(\mathrm{i}, \mathrm{j})$, "noise pixels" marked with $\mathrm{N}(\mathrm{i}, \mathrm{j})=0$ will be replaced by an estimated correction term. The NAFSM filter uses a square filtering window $\mathrm{W}_{2 s+1}(\mathrm{i}, \mathrm{j})$ with odd $(2 \mathrm{~s}+1) \mathrm{x}(2 \mathrm{~s}+1)$ dimensions, given as below

$$
\mathrm{W} 2 \mathrm{~s}+1(\mathrm{i}, \mathrm{j})=\{\mathrm{X}(\mathrm{i}+\mathrm{m}, \mathrm{j}+\mathrm{n})\}
$$

where $\mathrm{m}, \mathrm{n} \quad(-\mathrm{s}, \ldots, 0, \ldots, \mathrm{s})$.

In the filtering window $W 2 s+1(i, j)$, the number of noise free pixels $G 2 s+1(i, j)$ are counted using

$\mathrm{G} 2 \mathrm{~s}+1(\mathrm{i}, \mathrm{j})=\Sigma \mathrm{N}(\mathrm{i}+\mathrm{m}, \mathrm{j}+\mathrm{n})$, where $\mathrm{m}, \mathrm{n}(-\mathrm{s}, \ldots, 0, \ldots, \mathrm{s})$

The filtering window will be expanded by one pixel at each of its four sides (i.e., $s \leftarrow s+1$ ), until the criterion of $\mathrm{G} 2 \mathrm{~s}+1(\mathrm{i}, \mathrm{j})>1$ is achieved. These "noise-free pixels" are used for selecting the median pixel, $\mathrm{M}(\mathrm{i}, \mathrm{j})$, given by

$M(i, j)=\operatorname{median}\{X(i+m, j+n)\}$ with $N(i+m, j+n)=1$

Then , the absolute luminance difference $d(i, j)$ is computed:

$$
\mathrm{d}(\mathrm{i}+\mathrm{k}, \mathrm{j}+\mathrm{l})=|\mathrm{X}(\mathrm{i}+\mathrm{k}, \mathrm{j}+\mathrm{l})-\mathrm{X}(\mathrm{i}, \mathrm{j})|
$$

With $(\mathrm{i}+\mathrm{k}, \mathrm{j}+\mathrm{l}) \neq(\mathrm{i}, \mathrm{j})$

After that, the maximum absolute luminance difference is calculated in the $3 \times 3$ filtering window.

$\mathrm{D}(\mathrm{i}, \mathrm{j})=\max \{\mathrm{d}(\mathrm{i}+\mathrm{k}, \mathrm{j}+\mathrm{l})\}(9)$

Then the fuzzy reasoning is applied to $\mathrm{D}(\mathrm{i}, \mathrm{j})$.

$$
0 \text {, }
$$

$F(i, j)=$

$$
\text { (D(i,j)- T1)/( T2 - T2) }
$$

$$
\begin{aligned}
& : \mathrm{D}(\mathrm{i}, \mathrm{j})<\mathrm{T} 1 \\
& : \mathrm{T} 1 \leq \mathrm{D}(\mathrm{i}, \mathrm{j})<\mathrm{T} 2 \\
& : \mathrm{D}(\mathrm{i}, \mathrm{j})<\mathrm{T} 2
\end{aligned}
$$

where $\mathrm{D}(\mathrm{i}, \mathrm{j})$ is the local information used as input variable, and the two threshold values $\mathrm{T} 1$ and $\mathrm{T} 2$ are set to 10 and 30 , respectively, for optimal performance .

Lastly, the correction term for restoring corrupted pixels is computed by using the equation given below. 


$$
Y(i, j)=[1-F(i, j)] \cdot X(i, j)+F(i, j) \cdot M(i, j)
$$

\subsection{Adaptive Fuzzy Morphological Filtering of Impulse Noise in Images[2]}

In this method, a neural network implementation for fuzzy morphological operators is introduced, and by means of a training method and differentiable equivalent representations for the operators an efficient adaptation algorithms to optimize the structuring elements is derived.

Impulse noise commonly corrupts images either when defective sensors are used in the data acquisition, or when errors occur in the transmission.. Morphological filters are derived from a shape-oriented or structural approach to image processing. A binary or a multi-level image with these filters depends on the fitting of a function called structuring element (SE) to the image. Shape and support of SEs are usually chosen arbitrarily.

It is necessary that the SE be large enough to include the noise features and that its shape adapt to the geometry of the image features to be preserved.

To measure the performance of the filters, we use the peak signal-to-noise ratio (PSNR)

$P S N R=10 \log 10 \frac{\sum 255^{2}}{\sum(f(n)-g(n))^{2}}$

Where $f(n)$ and $g(n)$ correspond to the original and the filtered images.

Noise which does not fit within the optimal structuring element is effectively removed, while the image details are preserved.

TABLE 2 PSNR(dB) results of restored "Lena"image

\begin{tabular}{ccccc}
\hline \multirow{2}{*}{ Algorithm } & \multicolumn{3}{c}{ Positive Impulse Noise } \\
\cline { 2 - 5 } & & $10 \%$ & $20 \%$ & $30 \%$ \\
\hline \multicolumn{2}{c}{ Median filter } & 28.39 & 19.69 & 13.34 \\
\hline \multicolumn{2}{c}{ SD-ROM filter[27] } & 33.26 & 28.08 & 21.50 \\
\hline \multirow{2}{*}{ Opening filter } & $1 \times 1$ & 30.88 & 28.44 & 27.20 \\
(No. of blocks) & $2 \times 2$ & 31.54 & 29.59 & 27.70 \\
& $1 \times 4$ & 31.81 & 29.89 & 28.16 \\
& $16 \times 16$ & 32.62 & 30.55 & 28.76 \\
& $32 \times 32$ & 34.33 & 31.50 & 29.65 \\
& & & 32.61 & 30.71
\end{tabular}

the performance of the opening filter is improved by increasing the number of blocks. For severe noise conditions (20\% and $30 \%$ ), the opening filter gives considerably better performance than other methods.

The anti-extensive and extensive properties of fuzzy morphological operators indicate that filtering is more efficient with the opening and closing filters.

\subsection{Random- valued impulse noise removal using fuzzy weighted non-local means(FWNLM)[3]}

In this method, a new algorithm called the fuzzy weighted non-local means (FWNLM) filter for random valued impulse noise removal. An efficient fuzzy weighting function for the NLM algorithm is presented to shut off the impulsive components. The new filter processes pixels in accordance with the rule: the more a pixel is corrupted, the less the pixel is exploited to reconstruct image information. Experiments show that the FWNLM filter has a surprisingly good denoising capability.

The NLM algorithm has two drawbacks. The one is computationally expensive and many methods were proposed to accelerate it. The other is that it is sensitive to impulse noise. It cannot adequately remove impulse noise because NLM interprets the noisy pixels as image structures. 
TABLE 3 Results in PSNR after filtering images corrupted by mixed Gaussian and random- valued impulse noise

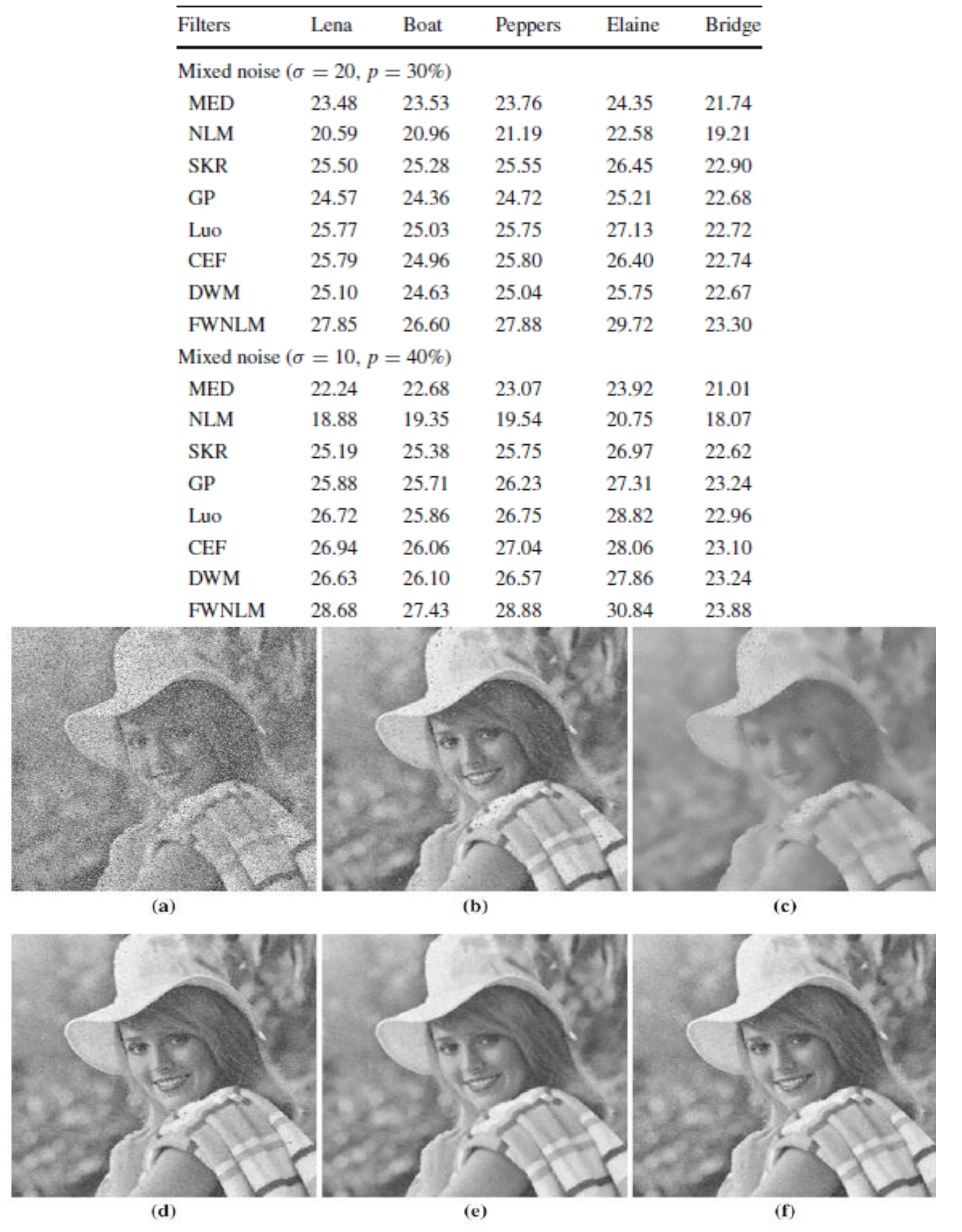




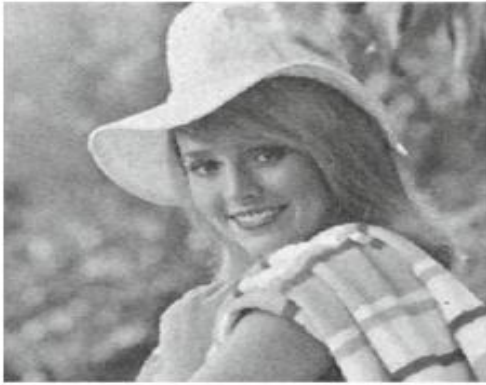

(g)

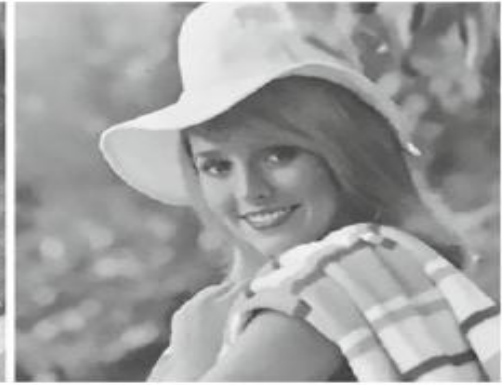

(h)

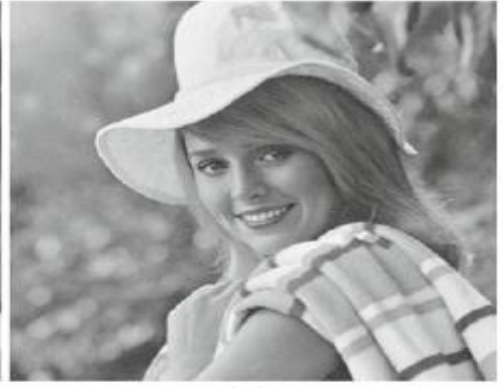

(i)

Figure 3 Results of different filters in restoring mixed noise $(\sigma=10, p=40 \%)$. a Noisy image, b SKR, c NLM, d GP, e Luo, f DWM, g CEF,h FWNLM and i the noise-free image

TABLE 4 Comparison of CPU time in seconds

\begin{tabular}{lrrrrr}
\hline & $p=40 \%$ & $p=50 \%$ & $p=60 \%$ & $\begin{array}{l}\sigma=20, \\
p=30 \%\end{array}$ & $\begin{array}{l}\sigma=10, \\
p=40 \%\end{array}$ \\
\hline MED & 0.1 & 0.1 & 0.1 & 0.1 & 0.1 \\
NLM & $1,117.6$ & $1,118.2$ & $1,117.7$ & $1,118.2$ & $1,118.2$ \\
SKR & $1,290.3$ & $1,452.1$ & $1,280.9$ & $1,272.6$ & $1,280.0$ \\
GP & 2.7 & 2.7 & 2.7 & 2.8 & 2.7 \\
Luo & 31.0 & 31.0 & 31.0 & 31.1 & 31.4 \\
CEF & 8.1 & 12.3 & 12.6 & 12.5 & 12.3 \\
DWM & 18.8 & 18.8 & 18.9 & 18.8 & 18.9 \\
FWNLM & $1,235.9$ & $1,269.5$ & $1,269.1$ & $1,269.1$ & $1,270.0$
\end{tabular}

performances of this filter are surprisingly satisfactory in terms of both visual quality and quantitative measurement. Moreover, this filter also can be used to remove mixed Gaussian and random-valued impulse noise.

\section{4 gradient detecting fuzzy logic-based algorithm (GDFF)[4]}

This method presents a gradient detecting fuzzy logic-based algorithm (GDFF) for image de-nosing issue. For the first step, GDFF selects different fixed filtering sub-windows to process the input signal by linear denoising. And then it modifies the de-noise results by a set of membership functions established by making full use of edge information. Finally, these signals are summed with weight to accomplish the image de-noising. Experiments illustrate that, GDFF performs a better de-noising effect with PSNR Gain 2.65-10.34dB compared with WFM and FIRE, when noise probability exceeds from 0.5 to 0.8 . Furthermore, GDFF exhibits more effective performance both in reserving image edge and in removing noise

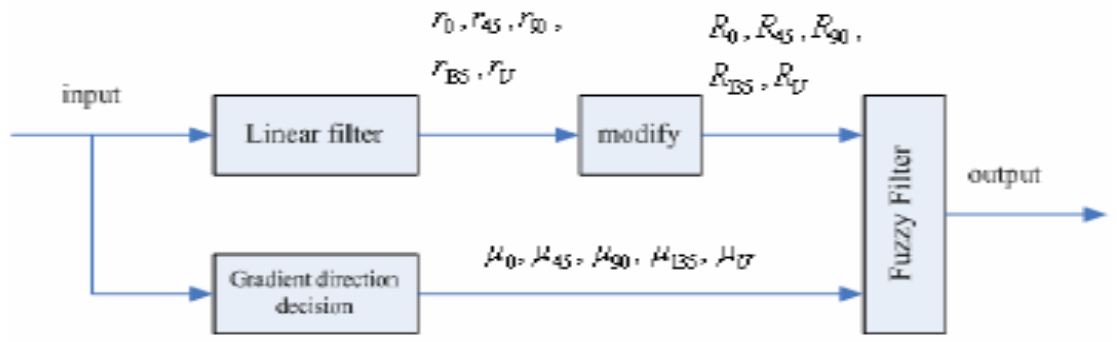

Figure 4 Flow chart of fuzzy logic-based de-noising algorithm based on gradient detecting

The outputs of Gradient direction decision are $\mu_{\mu_{0}, \mu_{45}, \mu_{30}, \mu_{15}, \mu_{07}}$ which estimate the degree of each pixel belongs to the edge direction according to the edge information. Linear filter's outputs are $r 0, r 90, r 45, r 135$, $r U$ which correspond to different directional models. Then, it modifies the de-noised result combining with the relativity of figure pels gray.

Finally, according to the output of judger, it sums the modified values with weight to accomplish the image de-noising adaptively. 


$$
P S N R=20 \log \left(\frac{255}{\sqrt{\frac{\sum[I 1(x ; y)-I 0(x, y)]^{2}}{x y}}}\right)
$$

$I O(x, y)$ is for original image, and

$I l(x, y)$ is de-noised one. $\mathrm{X}, \mathrm{Y}$ stands for numbers of pixel in rows and cols

TABLE 5 PSNR compare at the noise rate of $50 \%$ and $80 \%$

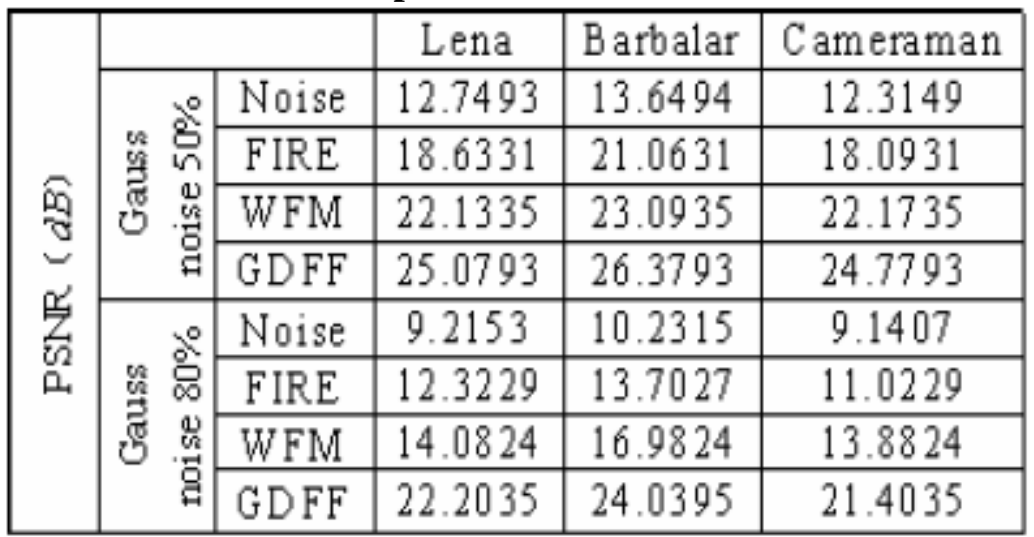

\subsection{Image Denoising Using Adaptive Neuro-fuzzy system[5]}

In this method, a generalized fuzzy inference system (GFIS) in noise image processing is proposed. The noisy image is divided by several $p \times p$ block. Each image block is fed into the GFIS model. The inferred output from the GFIS is compared with the noise-free image block.

Peak signal to noise ratio(PSNR)=

$$
10 \log 10 \frac{\sum 255^{2}}{1 / M N \sum\left(r_{\mathrm{i}}-x_{\mathrm{i}}\right)^{2}},
$$

Mean absolute error(MAE) $=1 / \mathrm{MN} \sum\left|r_{\mathrm{i}}-x_{\mathrm{i}}\right|$, Mean squad error(MSE)=1/MN $\sum\left(r_{\mathrm{i}}-x_{\mathrm{i}}\right)^{2}$

where $N \times M$ is the number of processed pixels and rij and $x i j$ denote the pixel values of the restored image and the original image, respectively.

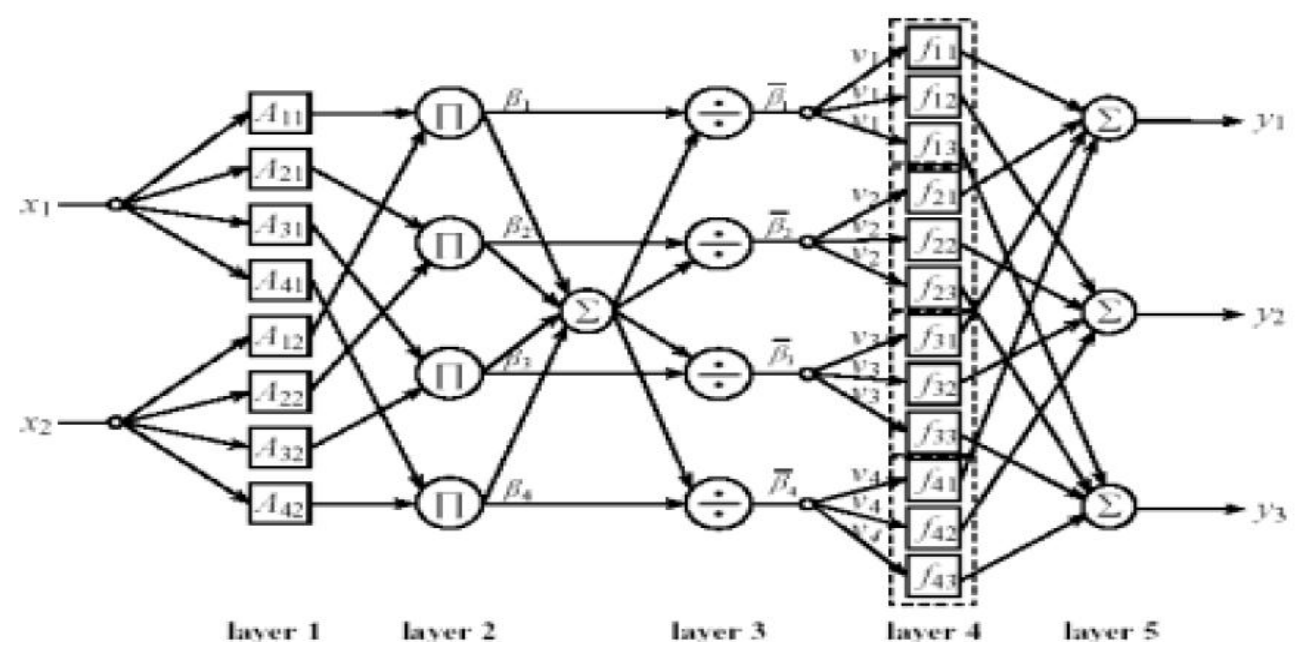

Figure 5 Schematic diagram of the generalized fuzzy inference model 


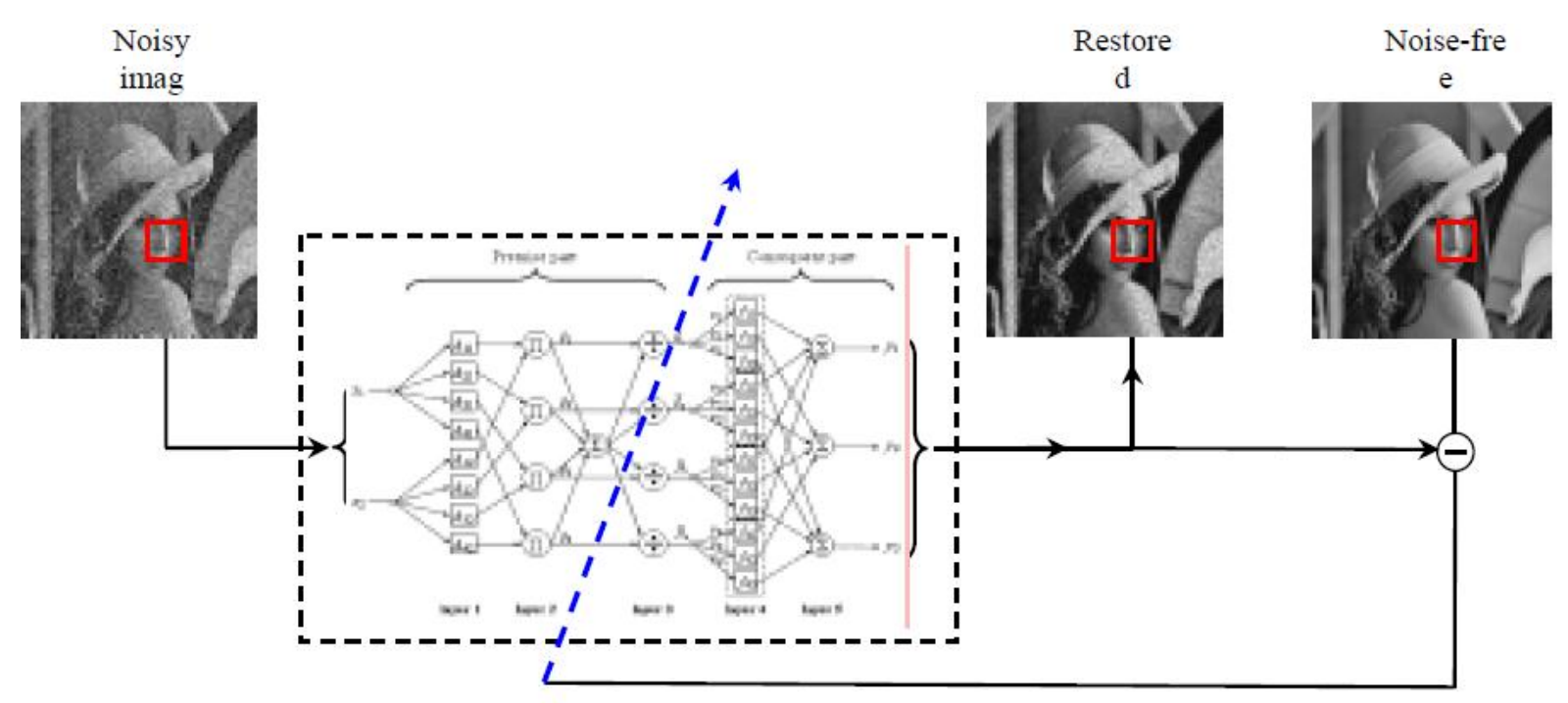

Figure 6 SCHEMATIC DIAGRAM FOR NOISE IMAGE PROCESSING
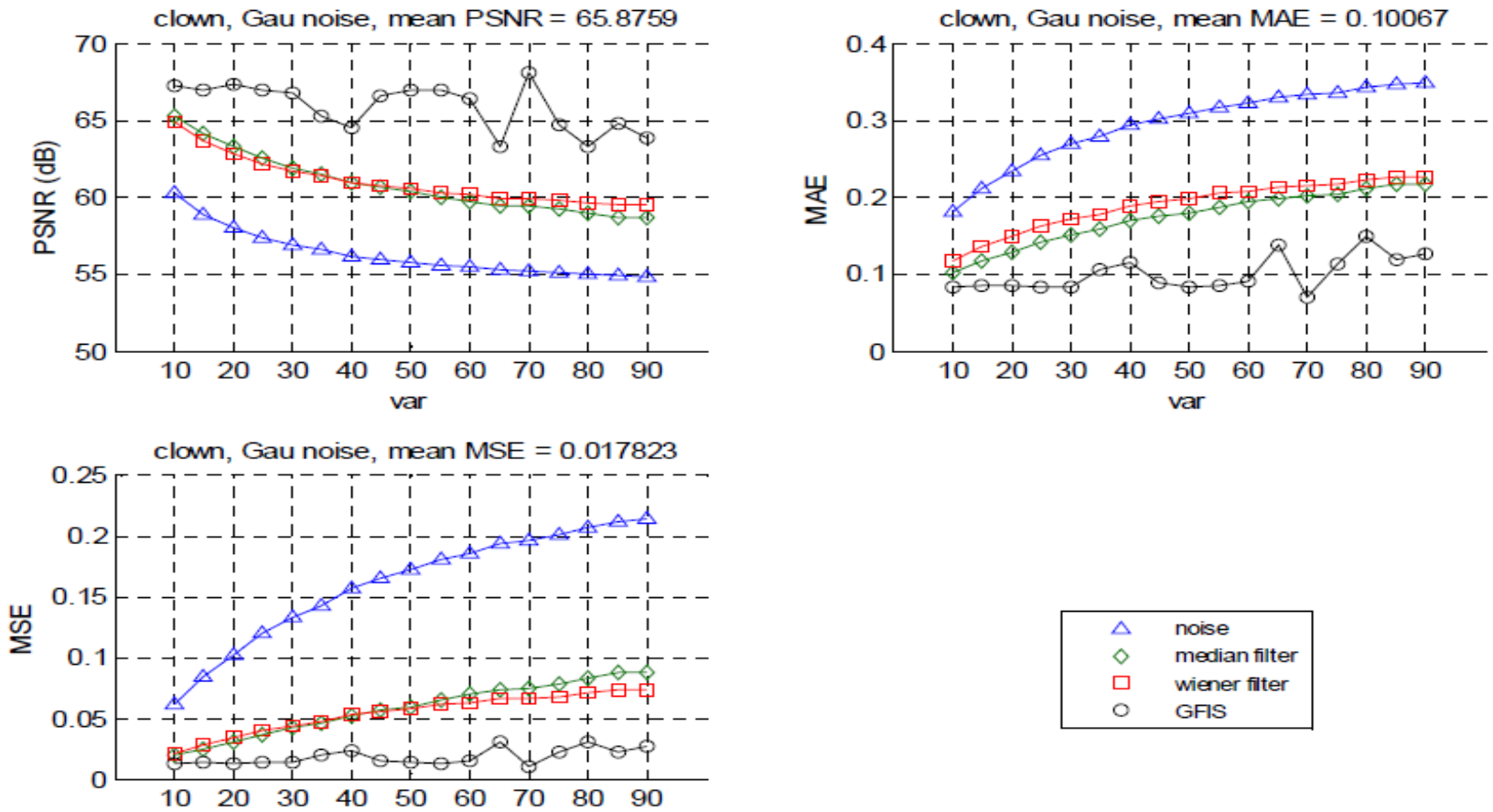

Figure 7 RESULTS IN PSNR,MAE AND MSE FOR THE CLOWN IMAGE AT VARIOUS VARIANCE LEVELS FOR MEDIAN FILTER, WIENER FILTER AND GFIS

GFIS gives the best performance in terms of noise suppression and detail preservation. The median filter and the wiener filter can achieve reasonably good performance for low corrupted images, but they will not work efficiently when the noise level is above $50 \%$.

\section{CONCLUSION}

Fuzzy image processing is the collection of all approaches that understand, represent and process the images, their segments and features as fuzzy sets. The representation and processing depend on the selected fuzzy technique and on the problem to be solved. Fuzzy logic is conceptually easy to understand. It is flexible. With any given system, it is easy to layer on more functionality without starting again from scratch. Fuzzy logic is tolerant of imprecise data. It can model nonlinear functions of arbitrary complexity.

Analysis of various fuzzy techniques gives the result that NAFSM is able to outperform FIDRM filter in terms of PSNR, execution time and MSE. The NAFSM filter is able to suppress high-density of salt-andpepper noise, at the same time preserving fine image details and edges. The NAFSM filter is able to yield good 
filtering results with efficient processing time even at high percentages of salt and pepper noise. Future research should focus on further shortening of the processing time of NAFSM filter by estimating the local information adaptively based on fuzzy inference. In the near future, we may extend GFIS to process color images. We can try to develop more adequate fuzzy sets by adaptively determining the shape and number of GDFF membership functions. In addition, different kinds of noise, such as pulse noise, salt pepper noise, can also be tested to evaluate the performance of GDFF further. Finally, we can add the implementation of a GDFF hardware version is in progress.

\section{REFERENCES}

[1] Stefan Schulte, Mike Nachtegael, Valérie De Witte, Dietrich Van der Weken, and Etienne E. Kerre, A Fuzzy Impulse Noise Detection and Reduction Method, IEEE Trans. Image Processing., vol. 15, no. 5,pp. 1153-1162, May 2006.

[2]. Jinsung Oh and Luis F. Chaparro, Adaptive Fuzzy Morphological Filtering of Impulse Noise in Images,,Multidimentional systems and signal processing,11,2000,pp.233-256.

[3] Jian $\mathrm{Wu} \cdot$ Chen Tang, Random-valued impulse noise removal using fuzzy weighted non-loca means,Springer-Verlog London Limited 2012, SIViP DOI 10.1007/s11760-012-0297-1.

[4] Liangrui Tang Hongting Wang Bing Qi, A New Fuzzy Logic Image De-noising Algorithm Based on Gradient Detection, IEEE Trans.,Fourth International Conference on Fuzzy Systems and Knowledge Discovery(FSKD 2007).

[5] Nguyen MinhThanh and Mu-Song Chen, Image Denoising Using Adaptive Neuro-fuzzy system, IAENG International Journal of Applied Mathematics, 36:1, IJAM_36_1_11,Advance Online Publication:1 February 2007.

[6] Zhang Xianzhong, Li Yaocheng, Jiang Lihui, Discussing and Comparing Lee Filter and Morphological Filtering Algorithm Using in Speckle Noise Reduction, LASER \&INFRARED, vol.31, no.2, pp.105-107,2001.

[7] J. H. Wang and W.J Liu., Histogram-Based Fuzzy Filter for Image Restoration, IEEE Trans. Syst., Man, Cybern part B, vol.32, no.2, pp.230-238, 2002.

[8] Frosio, I., Borghese, N.A., Statistical based impulsive noise removal in digital radiography. In: IEEE Trans. Med. Imaging 28(1), 3-16 (2009).

[9] Bovik, A., Handbook of Image and Video Processing. Academic, New York (2000).

[10] Xu, H., Zhu, G., Peng, H., Wang, D., Adaptive fuzzy switching filter for images corrupted by impulse noise. Pattern Recognit. Lett. 25, 1657-1663 (2004)

[11] Petrovic, N., Crnojevic, V., Universal impulse noise filter based on genetic programming. In: IEEE Trans. Image Process. 17(7), 1109-1120 (2008).

[12] Luo, W., An efficient algorithm for the removal of impulse noise from corrupted images. Int. J. Electron. Commun. 61, 551-555 (2007).

[13] Dong, Y., Xu, S., A new directional weighted median filter for removal of random-valued impulse noise. In: IEEE Signal Process.Lett. 14(3), 193-196 (2007).

[14] Ghanekar, U., Singh, A.K., Pandey, R., A contrast enhancementbased filter for removal of random valued impulse noise. In: IEEE Signal Process. Lett. 17(1), 47-50 (2010). 